\title{
Análise de preço e caracterização nutricional de alimentos ofertados em aplicativos de fast-food ao Teresinense em tempos de Covid-19
}

\author{
Price analysis and nutritional characterization of food offered in fast food applications to \\ Teresinense in Covid-19 times \\ Análisis de precios y caracterización nutricional de los alimentos oferecidos em aplicaciones de \\ alimentos rápidos a Teresinense em tempos de Covid-19
}

Recebido: 28/10/2021 | Revisado: 06/11/2021 | Aceito: 13/11/2021 | Publicado: 21/11/2021

\author{
Beatriz Figueredo da Silva \\ ORCID: https://orcid.org/0000-0003-3245-9863 \\ Centro Universitário Santo Agostinho, Brasil \\ E-mail: beatrizfsilva025@gmail.com \\ Ludimila Silva Lima \\ ORCID: https://orcid.org/0000-0002-6394-2308 \\ Centro Universitário Santo Agostinho, Brasil \\ E-mail: ludimilla.lima.2016@gmail.com \\ Luiza Marly Freitas de Carvalho \\ ORCID: https://orcid.org/0000-0002-6726-3994 \\ Centro Universitário Santo Agostinho, Brasil \\ E-mail: lumarnahid@gmail.com \\ Cicero Tadeu Tavares Duarte \\ ORCID: https://orcid.org/0000-0002-8565-1659 \\ Centro Universitário Santo Agostinho, Brasil \\ E-mail: cttduarte@gmail.com
}

\begin{abstract}
Resumo
O presente estudo teve como objetivo analisar o preço e a caracterização nutricional de alimentos ofertados em aplicativos de fast food na cidade de Teresina-PI em tempos de Covid-19. Foi realizado um estudo transversal, de natureza bibliográfica descritiva com abordagem quantitativa e qualitativa. Foram selecionados três aplicativos nomeados de A, B, e C. A coleta de dados foi realizada durante três (3) dias e se deu das $19 \mathrm{hrs} \mathrm{às} 22 \mathrm{hrs}$. Os dados foram contabilizados em planilha no software Excel, para o tratamento estatístico foi utilizado o programa Estat D+, e a apresentação dos resultados foram realizadas através de gráficos e tabelas do software Word. O aplicativo que apresentou a menor média de preço foi o APP-C (26,68 reais). Foram encontrados 148 estabelecimentos, destes foram coletadas 317 preparações e 1.899 ingredientes. Observou-se a alta demanda de ofertas de alimentos de baixa qualidade nutricional, ricos em carboidratos simples, gorduras e sódio. Conclui-se que os aplicativos analisados não incluem em suas ofertas às recomendações nutricionais e aporte calórico, verificando assim, negligências em algumas recomendações alimentares significativas, como incluir hortaliças, frutas e leguminosas nas preparações.
\end{abstract}

Palavras-chave: Covid-19; Alimentos; Fast-food.

\begin{abstract}
This study aimed to analyze the price and nutritional characterization of foods offered in fast food apps in the city of Teresina-PI in Covid-19. Was performed a cross-sectional study of descriptive bibliographic nature with a quantitative and qualitative approach. Three applications named A, B, and C were selected. Data collection was carried out during three (3) days and took place from $7 \mathrm{pm}$ to $10 \mathrm{pm}$. The data were recorded in a spreadsheet in the Excel software, the Stat D+ program was used for statistical treatment, and the presentation of the results was performed using graphics and tables in the Word software. The app with the lowest average price was APP-C (26.68 reais). 148 establishments were found, of which 317 preparations and 1,899 ingredients were collected. There was a high demand for food offerings of low nutritional quality, rich in simple carbohydrates, fats and sodium. It is concluded that the analyzed applications do not include nutritional recommendations and caloric intake in their offers, thus verifying, negligence in some significant dietary recommendations, such as including vegetables, fruits and legumes in the preparations.
\end{abstract}

Keywords: Covid-19; Foods; Fast-food.

\section{Resumen}

El presente estudio tuvo como objetivo analizar el precio y la caracterización nutricional de los alimentos ofrecidos en las aplicaciones de comida rápida en la ciudad de Teresina-PI en Covid-19. Fue realizado un estudio transversal de 
carácter descriptivo bibliográfico con un enfoque cuantitativo y cualitativo. Se seleccionaron tres aplicaciones denominadas A, B y C. La recolección de datos se realizó durante tres (3) días y se llevó a cabo de 19 a 22 horas. Los datos se registraron en una hoja de cálculo en el software Excel, se utilizó el programa Stat D + para el tratamiento estadístico y la presentación de los resultados se realizó mediante gráficos y tablas en el software Word. La aplicación con el precio promedio más bajo fue APP-C (26,68 reales). Se encontraron 148 establecimientos, de los cuales se recolectaron 317 preparados y 1.899 ingredientes. Hubo una gran demanda de alimentos de baja calidad nutricional, ricos en carbohidratos simples, grasas y sodio. Se concluye que las aplicaciones analizadas no incluyen recomendaciones nutricionales y aporte calórico en sus ofertas, verificando así, negligencia en algunas recomendaciones dietéticas significativas, como la inclusión de verduras, frutas y legumbres en las preparaciones.

Palabras clave: Covid-19; Alimentos; Fast-food.

\section{Introdução}

A alimentação é uma das atividades mais importantes do ser humano, incluindo fatores nutricionais e biológicos evidentes, bem como aspectos sociais, psicológicos e culturais relacionados ao ato de se alimentar. Portanto, esse fator abrange várias questões que vão desde a aquisição dos alimentos até a transformação dos mesmos em refeições (Melo; Calazans, 2018).

Uma boa nutrição é um fator significativo na determinação do estado de saúde e longevidade, e isso envolve a compreensão da importância de uma alimentação adequada e equilibrada (Lima, 2020).

A diversidade alimentar oriunda da sociedade moderna condiciona fortemente o comportamento, motivações e as escolhas alimentares. A disponibilidade e acessibilidade dos alimentos têm aumentado fazendo com que os indivíduos tenham cada vez mais oportunidades alimentares e consequentemente mais escolhas a fazer (Moraes, 2017).

A escolha alimentar tem relação com preferências correspondentes ao sabor dos alimentos e aos hábitos alimentares aprendidos desde a infância a partir do convívio social. O comportamento alimentar possui componentes fisiológicos e psicossociais, essas características influenciam as escolhas alimentares, formando um modelo alimentar, resultadas de uma soma de escolhas feitas por uma sociedade (Veiga; Melo, 2013).

O preço dos alimentos, de forma ainda mais evidente, se relaciona às chances de escolhas mais saudáveis ou não, uma vez que os alimentos de maior densidade energética são mais baratos que alimentos in natura (Moraes, 2017).

As profundas modificações sociais, econômicas e culturais que vêm ocorrendo no País, nas últimas décadas, levaram a alterações nos hábitos e comportamentos alimentares; por exemplo, o incremento da participação do consumo alimentar fora de casa, o aumento do consumo de alimentos processados e a substituição das refeições e preparações tradicionais por lanches com elevada concentração de energia, gorduras, açúcar de adição e sódio (Souza, 2013).

O consumo alimentar tem sofrido uma mudança na qualidade e quantidade dos produtos ofertados, gerando um consumo descontrolado de alimentos com alto teor de calórico, que associado ao sedentarismo, está acarretando uma geração com sobrepeso. Foram identificados, porém, fatores que colaboram de certa forma aos hábitos de consumo, como a renda e demanda, urbanização e globalização. As mudanças da dieta e da prática de atividades físicas podem ser atribuídas às mudanças demográficas e socioeconômicas (Moratoya, et al. 2013).

Segundo Verticchio (2020), no dia 30 de janeiro de 2020, a Organização Mundial de Saúde (OMS) declarou que o surto do novo Coronavírus se tornou uma emergência de saúde Pública de importância internacional e no dia 11 de março de 2020 foi caracterizada como pandemia. A Covid-19 é uma doença causada pelo Coronavírus, denominada SARS-CoV-2, que apresenta um espectro clínico variando de infecções assintomáticas a quadros graves, e seus sintomas podem variar de um resfriado, a uma Síndrome Gripal-SG até uma pneumonia severa (Brasil, 2020).

No Brasil, a identificação do primeiro caso da Covid-19 ocorreu em 26 de fevereiro de 2020 e a resposta da Secretaria de Vigilância em Saúde e do Ministério da Saúde (SVS/MS) à epidemia foi imediata, onde foram iniciadas diversas ações para a prevenção e enfretamento do vírus, dentre eles a recomendação do isolamento social (Croda; Garcia, 2020). Com isso, 
atividades que faziam parte da rotina da população foram interrompidas principalmente as relacionadas às relações humanas, forçando a necessidade de novas adaptações para as quais muitas pessoas ainda não estavam preparadas (Ribeiro, et al., 2020).

O confinamento impôs, inevitavelmente, uma alteração dos comportamentos, acarretando desafios físicos e psicológicos. O isolamento físico das relações sociais, aliado a uma inquietação geral com a saúde e com as questões econômicas, gerou níveis mais elevados de estresse e ansiedade. O estresse emocional associa-se à adoção de determinados comportamentos alimentares e pode conduzir a alterações dos hábitos alimentares em contextos de isolamento social (Ferreira, 2020).

Diante das mudanças de hábitos dos consumidores e da necessidade de maior praticidade para se alimentar, um mercado que vem se destacando é o de serviços online de alimentação (Stecca, 2020).

O fast food é classificado como uma alimentação industrializada, quase sempre muito calórica que se caracteriza tanto pela comida quanto pelo serviço rápido, eficiente, impessoal, padronizada. A preparação do tipo fast food é considerada uma alimentação de risco para a saúde ou no mínimo como hábito alimentar inadequado (Freitas; Oliveira, 2008).

Os serviços online de alimentação estão a revolucionar o conceito de entrega de comida (Marques, 2019). Consumidores que fazem uso de aplicativos de delivery de comida buscam praticidade, comodidade e rapidez ao pedir seus alimentos, escolhendo o aplicativo por sua credibilidade, facilidade de uso e pela variedade de restaurantes próximos que encontram no mesmo, podendo-se citar, ainda com clareza das informações (Ponte, 2016).

$\mathrm{O}$ controle dos custos tem sido um instrumento utilizado para manter empresas competitivas diante da concorrência e melhorar sua relação com consumidores por meio de preços mais atrativos. O gestor ou gerente é o profissional que tem a responsabilidade de maximizar a satisfação das partes envolvidas nos negócios, otimizando a utilização de recursos na produção de serviços e/ou produtos que negocia (Melo; Calazans, 2018).

Na maioria dos aplicativos, há a possibilidade do usuário permita o uso de sua localização e, a partir dessa informação, os aplicativos sugerem ao cliente uma lista com os restaurantes mais próximos a ele. Ainda na maioria dos referidos aplicativos, é possível que o consumidor ative as notificações, meio pelo qual o aplicativo passa a avisá-lo do status do seu pedido e da divulgação de promoções e sugestões de pedidos (Firmo; Cunha, 2020).

Os gestores dos aplicativos obtêm informações sobre o perfil do consumidor, podendo utilizá-las de forma a delinear o comportamento alimentar do cliente, oferecendo-lhe produtos que mais se assemelham ao seu perfil, incentivando o consumo sem se preocupar com uma alimentação adequada. Em contrapartida, algumas empresas têm apostado na entrega de comida saudável a fim de atender diferentes nichos, tais como opções veganas, fitness ou pratos feitos, dentre outros, com o intuito de se diferenciar nesse setor (Firmo; Cunha, 2020).

Pelo fato de ser um tema atual, onde o isolamento social é vivenciado em todo o mundo o que afeta consequentemente o comportamento alimentar, implicando assim em escolhas alimentares com um baixo nível de saudabilidade, o presente estudo teve como objetivo realizar a análise de preço e caracterização dos alimentos ofertados em aplicativos de fast food ao Teresinense em tempos de Covid-19.

\section{Metodologia}

Trata-se de um estudo transversal, de natureza bibliográfica descritiva com abordagem quantitativa e qualitativa. Segundo Estrela, C. (2018), o estudo transversal é um procedimento que tem como intuito avaliar amostras representativas e aleatórias da população, independentemente da existência da exposição. A busca dos dados foi realizada a partir de determinadas empresas no ramo da alimentação, com intenção de analisar o preço e caracterização de alimentos ofertados em aplicativos de fast food ao teresinense em tempos de Covid-19.

A pesquisa foi realizada na cidade de Teresina-PI, os dados foram coletados nos dias 22, 23 e 24 de abril de 2021. Primeiramente com um suporte de dois celulares foi realizado o acesso gratuito nas redes de aplicativos de fast food, em seguida 
foram selecionados três (3) aplicativos nomeados de A, B e C utilizados pela população Teresinense. Ao acessar a rede de aplicativos de fast food pelo celular dia 22/04/2021 às 19 horas, foram disponibilizados cardápios online com ofertas de preparações contendo anúncios do tipo: (ofertas imperdíveis até 20\%, show de descontos até 70\%, Fim de semana com churrasco, imperdíveis por 5,00 reais, jantar barato a partir de 10 reais, bom e barato 10 reais, ofertas saudáveis) junto ao preço e restaurantes para os aplicativos A, B e C. Durante todo o processo de análise todas as preparações ofertadas foram descritas em uma tabela, contabilizando os ingredientes, preços e restaurantes, finalizando as 22:00 horas. Foi realizado o mesmo método nos aplicativos $\mathrm{A}, \mathrm{B}$ e C durante os dias 22/04/2021 (quinta-feira), 23/04/2021 (sexta-feira) e 24/04/2021 (sábado), no horário 19:00 às 22:00 horas. Todas as ofertas foram descritas em uma tabela estruturada, contendo nome do aplicativo, data e restaurante selecionado, contabilizando assim os ingredientes e preços de cada noite.

Utilizaram-se os seguintes critérios de inclusão para a seleção: Somente aplicativos que disponibilizam o cardápio online com ingredientes e preparações com preços de oferta. E excluiu-se da pesquisa empresas cadastradas nos aplicativos que não se enquadrem no ramo de alimentação, como depósito de bebidas.

Os dados foram contabilizados em planilha no software Excel (2019). Para o tratamento estatístico foi utilizado o programa Estat D+(1998), e a apresentação dos resultados foi através de gráficos e tabelas do software Word (2019).

O EstatD+ é um aplicativo de estatística descritiva pode ser considerada como um conjunto de técnicas analíticas utilizado para resumir o conjunto dos dados recolhidos numa dada investigação, que são organizados, geralmente, através de números, tabelas e gráficos. Pretende proporcionar relatórios que apresentem informações sobre a tendência central e a dispersão dos dados. Para tal, deve-se evidenciar: valor mínimo, valor máximo, soma dos valores, contagens, média, moda, mediana, variância e desvio padrão (Morais, 2005).

\section{Resultados e Discussão}

Respeitando os critérios de inclusão e exclusão, foram definidos três (03) aplicativos de fast food que disponibilizam preparações em ofertas, estes foram classificados em A, B e C. Os aplicativos de fast food delivery pesquisados são compostos por restaurantes, lanchonetes, padarias, hamburguerias e pizzarias.

Obteve-se o resultado de 148 estabelecimentos em funcionamento no horário da coleta de dados, destes foram coletadas 317 preparações e a totalidade de 1.899 ingredientes. A média do total de preços extraídos foi 32,49 reais por preparação.

No Gráfico 1, ficou evidenciado que o aplicativo C compreende a menor média de preço 26,68 reais nos três (03) dias de coleta de dados das preparações analisadas, seguido do aplicativo B com a média de preço de 33,88 reais por preparação, e o aplicativo com a maior média das preparações foi o aplicativo A, onde o preço médio foi de 36,93 reais. 
Gráfico 1. Média de preço diário das preparações ofertada em aplicativos de fast food na cidade de Teresina/PI.

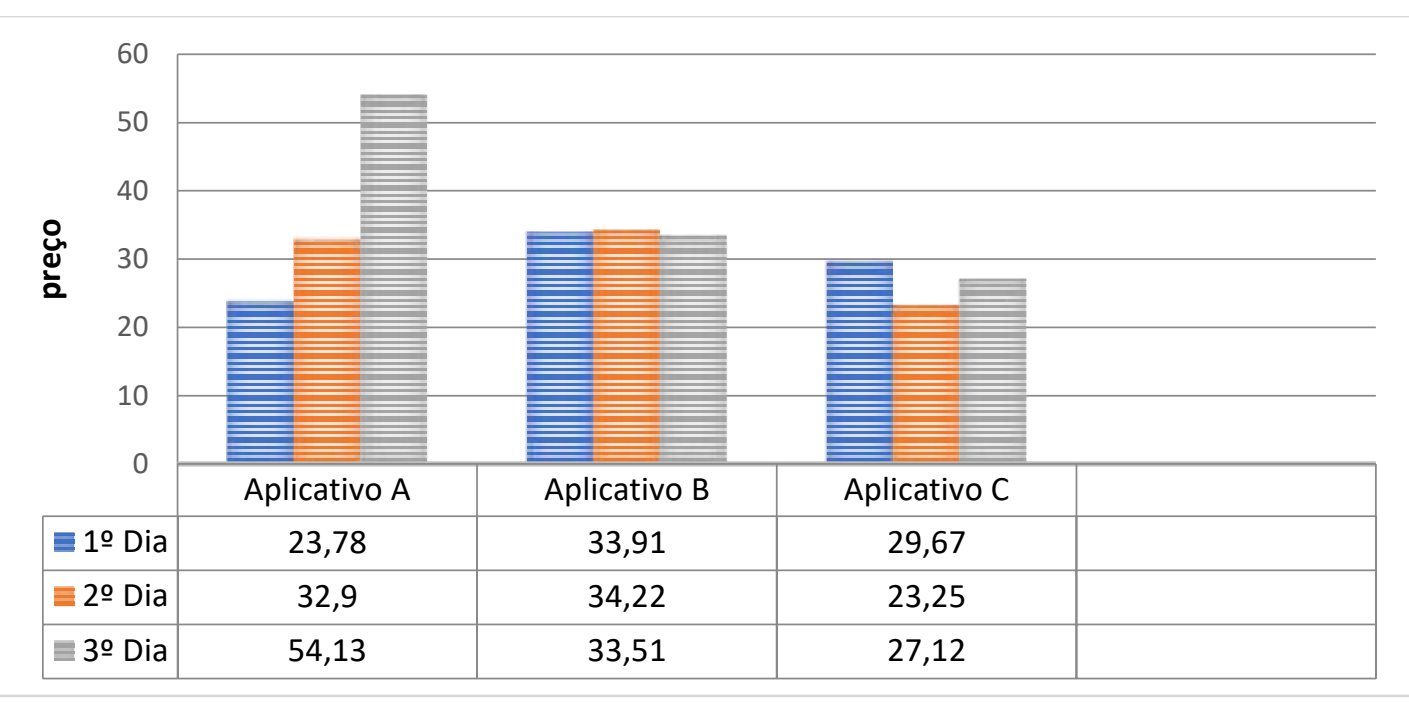

Fonte: Lima, L.; Silva, B. (2021).

Segundo Zielke (2010), o preço é um elemento importante na escolha de uma loja de alimentos. O domínio do melhor preço em relação aos concorrentes é um dever essencial para os gerentes que atuam na criação de estratégias de precificação (Panizzon, et al., 2013).

De acordo com Panizzon (2013) a imagem de preço pode ser definida como uma variável latente multidimensional que consiste em crenças e sentimentos subjetivos (armazenados na memória do consumidor), sobre as atividades de preço de uma empresa, loja ou categoria. Baseado nestas crenças e sentimentos os consumidores podem derivar uma avaliação geral da política de preço que pode ser rotulada de "atitude de preço", e que está fortemente associada ao comportamento de compra

Em relação às preparações ofertadas nos aplicativos, observou-se uma maior prevalência de oferta da preparação burger (45\%), seguido da pizza (20\%), refeições (19\%), hot-dog (12\%), tapioca (3\%) e cuscuz (1\%) conforme descrito no Gráfico 2.

Gráfico 2. Preparações ofertadas em aplicativos de fast food na cidade de Teresina-PI.

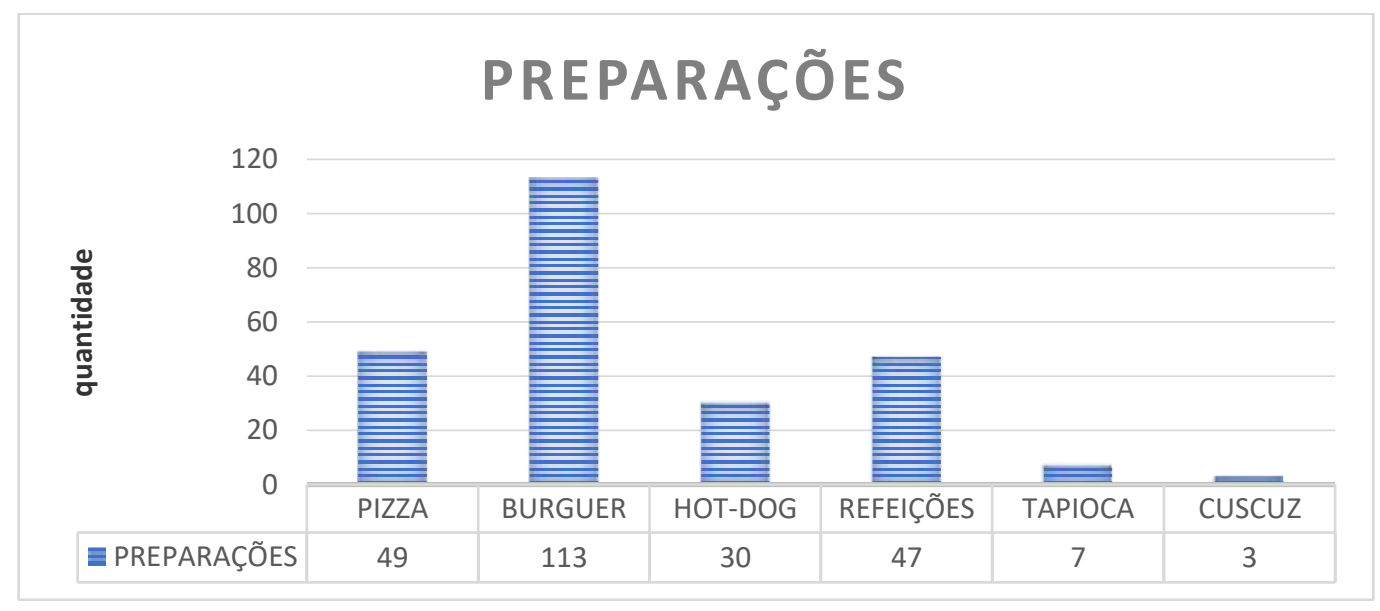

Fonte: Lima, L.; Silva, B., (2021).

Fórmulas rápidas de comer representada pelo hambúrguer, a pizza e o hot-dog passam a constituir um conjunto alimentar de consumo em massa, impondo um novo sabor e causando uma verdadeira ruptura no comer tradicional, trazendo no seu bojo mudanças nos hábitos e práticas alimentares. No Brasil, um novo padrão alimentar está se delineando, com prejuízos dos produtos da dieta tradicional do povo. O arroz, feijão, a farinha de mandioca, que foram, desde o século XVIII, a base do 
cardápio da maioria da população, perdem cada vez mais espaço para os produtos industrializados e com maior valor agregado (Santos, 2006).

$\mathrm{Na}$ Tabela 1 estão descritas a frequência dos alimentos ou ingredientes presentes nas preparações ofertadas nos aplicativos de fast food em ordem decrescente.

De uma somatória de 1.899 ingredientes, o de maior contribuição foi o queijo, totalizando 12,63\% dos ingredientes presente em 240 pratos diferentes, associadamente ao queijo vem o requeijão, produto presente em 18 preparações distintas representando $0,94 \%$ dos ingredientes de preparações ofertadas.

O tomate apresentou frequência de 8,63\% nas preparações, ou seja, apareceu em 164 pratos diferentes, o pão apresentou 7,53\% do total de ingredientes e 143 do total de preparações analisadas e o hamburguer representou 5,95\% da quantidade de ingredientes, estando presente em 113 preparações (tabela 1).

Os alimentos in natura ou minimamente processados presente nas preparações ofertadas são: tomate, cebola, folhagens, frango, ovo, especiarias, carne, arroz, suco natural, pimentão, macaxeira, macarrão, batata, camarão, peixe, cenoura, feijão, tapioca, beterraba, brócolis, repolho, abacaxi, arroz integral, banana, batata doce, o suco natural, massa de sêmola, nata, canela, nozes e pepino. A quantidade de alimentos in natura ou minimamente processados disponíveis nas preparações de fast food foi de $45,81 \%$ do total de ingredientes (tabela 1 ).

Os alimentos processados presentes nas preparações ofertadas são: queijo, pão, massa de pizza, milho, farofa, azeitona, requeijão, ervilha e o cuscuz que correspondem a 30,23\% do total de ingredientes (Tabela 1).

Já os ultraprocessados como o hambúrguer, bacon, presunto, batata-palha, molhos, calabresa, salsicha, batatas-fritas, geléias, creme de leite, salame, granola, fazem parte da dieta de teresinenses que aderem produtos de fast food delivery com preços de oferta. A quantidade de alimentos ultraprocessados presente nas preparações analisadas é de $25,91 \%$ da totalidade de ingredientes (Tabela 1).

Tabela 1. Alimentos presentes nas preparações ofertadas em aplicativos de fast food na cidade de Teresina-PI.

\begin{tabular}{|c|c|c|c|c|c|}
\hline \multirow[t]{2}{*}{ ALIMENTOS } & \multicolumn{3}{|c|}{ QUANTIDADE } & \multirow[t]{2}{*}{ TOTAL } & \multirow[t]{2}{*}{$(\%)$} \\
\hline & $A P P-A$ & $A P P-B$ & $A P P-C$ & & \\
\hline Queijo & 100 & 57 & 83 & 240 & $12,63 \%$ \\
\hline Tomate & 73 & 40 & 51 & 164 & $8,63 \%$ \\
\hline Pão & 58 & 47 & 38 & 143 & $7,53 \%$ \\
\hline Cebola & 66 & 37 & 29 & 132 & $6,95 \%$ \\
\hline Folhagens & 50 & 24 & 40 & 114 & $6,0 \%$ \\
\hline Hamburguer & 45 & 32 & 36 & 113 & $5,95 \%$ \\
\hline Bacon & 25 & 33 & 24 & 82 & $4,31 \%$ \\
\hline Presunto & 45 & 19 & 05 & 69 & $3,63 \%$ \\
\hline Batata-palha & 40 & 16 & 07 & 63 & $3,31 \%$ \\
\hline Frango & 23 & 18 & 22 & 63 & $3,31 \%$ \\
\hline Ovo & 39 & 16 & 07 & 62 & $3,26 \%$ \\
\hline Molhos & 17 & 25 & 15 & 57 & $3,0 \%$ \\
\hline Especiarias & 14 & 13 & 27 & 54 & $2,84 \%$ \\
\hline Carne & 28 & 18 & 6 & 52 & $2,73 \%$ \\
\hline Massa para pizza & 04 & 15 & 30 & 49 & $2,58 \%$ \\
\hline Arroz Branco & 32 & 12 & 03 & 47 & $2,47 \%$ \\
\hline Milho & 18 & 19 & 10 & 47 & $2,47 \%$ \\
\hline
\end{tabular}


Research, Society and Development, v. 10, n. 15, e129101522475, 2021

(CC BY 4.0) | ISSN 2525-3409 | DOI: http://dx.doi.org/10.33448/rsd-v10i15.22475

\begin{tabular}{|c|c|c|c|c|c|}
\hline Calabresa & 17 & 10 & 19 & 46 & $2,42 \%$ \\
\hline Suco natural & 28 & 12 & 00 & 40 & $2,10 \%$ \\
\hline Farofa & 17 & 09 & 08 & 34 & $1,79 \%$ \\
\hline Azeitona & 04 & 09 & 17 & 30 & $1,57 \%$ \\
\hline Salsicha & 13 & 15 & 02 & 30 & $1,57 \%$ \\
\hline Pimentão & 25 & 03 & 01 & 29 & $1,52 \%$ \\
\hline Fritas & 05 & 08 & 06 & 19 & $1,0 \%$ \\
\hline Requeijão & 05 & 09 & 04 & 18 & $0,94 \%$ \\
\hline Macaxeira & 09 & 06 & 01 & 16 & $0,84 \%$ \\
\hline Ervilha & 10 & 00 & 01 & 11 & $0,57 \%$ \\
\hline Macarrão & 10 & 00 & 01 & 11 & $0,57 \%$ \\
\hline Batata & 05 & 03 & 02 & 10 & $0,52 \%$ \\
\hline Camarão & 09 & 00 & 00 & 09 & $0,47 \%$ \\
\hline Peixe & 06 & 03 & 00 & 09 & $0,47 \%$ \\
\hline Cenoura & 03 & 03 & 02 & 08 & $0,42 \%$ \\
\hline Feijão & 07 & 00 & 00 & 07 & $0,36 \%$ \\
\hline Geleias & 00 & 00 & 07 & 07 & $0,36 \%$ \\
\hline Tapioca & 07 & 00 & 00 & 07 & $0,36 \%$ \\
\hline Pão de alho & 06 & 00 & 00 & 06 & $0,31 \%$ \\
\hline Beterraba & 05 & 00 & 00 & 05 & $0,26 \%$ \\
\hline Brócolis & 02 & 03 & 00 & 05 & $0,26 \%$ \\
\hline Repolho & 05 & 00 & 00 & 05 & $0,26 \%$ \\
\hline Abacaxi & 00 & 00 & 04 & 04 & $0,21 \%$ \\
\hline Creme de leite & 02 & 00 & 02 & 04 & $0,21 \%$ \\
\hline Picles & 00 & 04 & 00 & 04 & $0,21 \%$ \\
\hline Arroz Integral & 00 & 03 & 00 & 03 & $0,15 \%$ \\
\hline Banana & 01 & 00 & 02 & 03 & $0,15 \%$ \\
\hline Batata doce & 00 & 03 & 00 & 03 & $0,15 \%$ \\
\hline Cuscuz & 03 & 00 & 00 & 03 & $0,15 \%$ \\
\hline Suco detox & 00 & 03 & 00 & 03 & $0,15 \%$ \\
\hline Massa de sêmola & 02 & 00 & 00 & 02 & $0,10 \%$ \\
\hline Nata & 02 & 00 & 00 & 02 & $0,10 \%$ \\
\hline Salame & 00 & 00 & 02 & 02 & $0,10 \%$ \\
\hline Canela & 00 & 00 & 01 & 01 & $0,05 \%$ \\
\hline Granola & 01 & 00 & 00 & 01 & $0,05 \%$ \\
\hline Nozes & 01 & 00 & 00 & 01 & $0,05 \%$ \\
\hline Pepino & 01 & 00 & 00 & 01 & $0,05 \%$ \\
\hline
\end{tabular}

Fonte: Lima, L.; Silva, B., (2021).

O queijo é uma das formas mais antigas de alimentos industrializados (WALTHER et. al., 2008), é um concentrado lácteo constituído de proteínas, lipídeos, carboidratos, sais minerais, cálcio, fósforo e vitaminas, entre elas A e B e é um dos produtos mais consumidos no Brasil (Perry, 2003; Altissimo \& Junkerfuerbom, 2016).

O tomate é rico em vitaminas, aminoácidos essenciais, açucares, fibras dietéticas e apresentam grandes quantidades de vitamina B e C, ferro e fósforo. Os frutos podem ser consumidos frescos, cozidos, em saladas, molhos e preparações como carne 
e peixe (Naika, et al. 2006). Bertotti e Massuquetti (2010) afirmam que o preço do fruto é um fator determinante para o consumo do produto.

Hamburguer entende-se por um produto cárneo, obtido de carne de açougue, adicionado ou não de gordura e ingredientes, moldado e submetido ao processo tecnológico adequado. Os requisitos das características sensoriais do hamburguer envolvem textura, cor, sabor e odor próprios, podendo ter no máximo $23 \%$ de gordura e no mínimo $15 \%$ de proteína (Brasil, 2000). Já Romans et al. (1985) elucida hambúrguer como um bife de carne moída, fresco ou congelado, com ou sem adição de gordura e/ou condimentos, que não deve apresentar mais de 30\% de gordura e não deve conter adição de água.

O pão é considerado o alimento processado mais antigo sendo consumido rotineiramente por quase todo o mundo (Silva, 2018), nos últimos anos o consumo de pão vem crescendo em decorrência do aumento de poder aquisitivo da população, principalmente aquela parcela que até então estava excluída e passou a consumir produtos industrializados (Cunha, 2012).

Os alimentos in natura são adquiridos diretamente de plantas ou de animais e não sofrem qualquer alteração após deixar a natureza. Já os alimentos minimamente processados correspondem a alimentos in natura que foram expostos a processos de limpeza, extração de partes não comestíveis ou indesejáveis, fracionamento, moagem, secagem, fermentação, pasteurização, refrigeração, congelamento e processos similares que não envolvam agregação de sal, açúcar, óleos gorduras, ou outras substâncias ao alimento original (Brasil, 2014).

Os alimentos processados são produtos alimentares que contém vários ingredientes, e que passam por diversos processos industriais, contribuindo para um perfil nutricional não balanceado, pois são produtos com grandes quantidades de carboidratos refinados, gorduras saturadas, sódio e baixo teor em fibras (Carvalho, 2020).

Os alimentos ultraprocessados são formulações industriais feitas completamente ou predominantemente de substâncias extraídas de alimentos (óleos, gorduras, açúcar, amido, proteínas), derivadas de partes de alimentos (gorduras hidrogenadas, amido modificado) ou sintetizadas em laboratório com base em matérias orgânicas como petróleo e carvão (corantes, aromatizantes, realçadores de sabor e vários tipos de aditivos usados para dotar os produtos de propriedades sensoriais atraentes), (Brasil, 2014)

Verifica-se que apesar dos alimentos in natura ou minimamente processados apresentarem uma porcentagem de 45,81\% do total de ingredientes, os alimentos processados e ultraprocessados se sobressaem na dieta do teresinense que faz uso de aplicativos de fast food, totalizando 54,19\% dos ingredientes. O Guia Alimentar para População Brasileira recomenda dar preferência sempre a alimentos in natura ou minimamente processados e preparações culinárias á alimentos ultraprocessados, devido a seus ingredientes, alimentos ultraprocessados são nutricionalmente desbalanceados (Brasil, 2014).

Conforme Oliveira e Freitas (2008), os alimentos do tipo fast food apresentam preço acessível, menor tempo entrega e são amplamente consumidos nos dias atuais, pois possui uma capacidade de "adaptação" a culturas diversas e a gostos locais, fazendo assim com que o produto atenda ao paladar dos indivíduos que se podem mesclar diversos ingredientes na sua preparação contribuindo para que esse produto se torne sucesso em todo mundo (Rodrigues, et al., 2019; Lopes, et al., 2019). Trata-se de uma alimentação normalmente com um aporte calórico elevado, rica em lipídeos, carboidratos e sódio, pobre em vitaminas, minerais e fibras alimentares.

\section{Conclusão}

Este estudo teve como objetivo analisar o preço e caracterização de alimentos ofertados em aplicativos de fast food ao teresinense em tempos de Covid-19. Durante o período de restrições e isolamentos exigidos pela pandemia do Covid-19 a sociedade tem sofrido mudanças significativas em seus hábitos alimentares e como isso a diversidade alimentar tem influenciado de forma direta, pois a sociedade motiva fortemente o comportamento e as escolhas alimentares, incluindo alguns fatores como: fácil acesso, marketing, e preços acessíveis. Entre os achados mais preeminentes, ficou evidenciado que os aplicativos de fast 
food apresentam preços de oferta das mais variadas preparações para fim de ganhar todos os tipos de consumidores, a publicidade vem influenciando o crescimento de vendas durante o isolamento social, aumentando a demanda de refeições caracterizadas por apresentarem uma baixa qualidade nutricional, alto teor de carboidratos simples, gorduras e sódio.

Conclui-se que as preparações analisadas se consumidas frequentemente podem trazer consequências a saúde, tornandose um dos principais fatores para doenças crônicas como diabetes, hipertensão e obesidade, pois aplicativos analisados não incluem em suas ofertas recomendações nutricionais e aporte calórico para tingir as necessidades nutricionais, verificando-se negligências em algumas recomendações alimentares significativas, como incluir hortaliças, frutas e leguminosas nas preparações, visando assim melhorar a saúde e bem estar do consumidor. O Guia Alimentar orienta que os alimentos in natura ou minimamente processados, em grande variedade e predominantemente de origem vegetal, são a base para uma alimentação nutricionalmente balanceada, saborosa, culturalmente apropriada e promotora de um sistema alimentar socialmente e ambientalmente sustentável.

Apesar da expressiva importância do tema, pelo fato de ser um assunto altamente prevalente no mundo, e de tamanha importância para o meio cientifico, ainda se fazem necessários futuros estudos para verificar de forma mais precisa a qualidade dos alimentos ofertados nos aplicativos de fast-food e o impacto na saúde dos consumidores. Espera-se com esse estudo despertar interesse pela temática, contribuindo assim para pesquisas posteriores de larga escala em relação à análise de preço e caracterização nutricional de alimentos ofertados em aplicativos de fast food ao Teresinense em tempos de Covid-19, para que ocorra o desenvolvimento de novos artigos, com finalidade de fornecer informações mais consistentes sobre o tema, gerando condutas mais aprimoradas e especificas a população que desfrutam desses aplicativos de fast food em seu dia a dia, possibilitando orientações nutricionais direcionadas com bases cientificas.

\section{Referências}

Altissimo, C. V., \& Junkerfeurbom, F. D. (2016). Desenvolvimento do plano APPCC para linha de produção de queijo mussarela (Bachelor's thesis, Universidade Tecnológica Federal do Paraná).

Bertotti, G., \& Massuquetti, A. (2010). Determinantes da oferta e da demanda de tomate no Brasil, de 1994 a 2008 . Revista de Política Agrícola, 19(4), 39-49.

Costa, A., Bueno, P. K. T., \& Ferreira, M. C. M. (2020). Pesquisa de coliformes em hambúrgueres artesanais comercializados em Campo Mourão, Brasil. SaBiosRevista de Saúde e Biologia, 15(2), 11-15.

Brasil, Ministério da Saúde (2020). Coronavírus Brasil. https://covid.saude.gov.br

Brasil. (2014). Ministério da Saúde. Guia alimentar para a população brasileira. (2a ed.).

Carvalho, V. N., Couto, A. N., Vitiello, I. P., Severgnini, C., \& Pohl, H. H. (2020). Consumption of industrialized and in natura foods by adults and its relationship to the nutritional state/consumo de alimentos processados/ultraprocessados e in natura por adultos e sua relacao com o estado nutricionaL. Revista Brasileira de Obesidade, Nutrição e Emagrecimento, 14(84), 66-73.

Castro, N. S., de Bittencourt, J. A., Chaves, F. A. V., Barreiro, J. H. L. C. D., \& Reis, C. V. S. (2015). A influência do marketing digital sobre a escolha dos consumidores. XII Simpósio de Excelência em Gestão e Tecnologia-SEGeT. Rio de Janeiro, Brazil. Retrieved from https://www. aedb. br/seget/artigos2015. php.

Croda, J. H. R., \& Garcia, L. P. (2020). Resposta imediata da Vigilância em Saúde à epidemia da Covid-19.

Cunha, A. O. D. (2012). Cadeia produtiva do pão: fontes informacionais utilizadas no planejamento de novos produtos.

Dam, B. V., Goffau, M. D., Lidth de Jeude, J. V., \& Naika, S. (2006). A cultura do tomate: produção, processamento e comercialização. Agrodok.

Estrela, C. (2018). Metodologia científica: ciência, ensino, pesquisa. Artes Médicas.

Fátima Vestena, R., Scremin, G., \& Bastos, G. D. (2018). Alimentação saudável: contribuições de uma sequência didática interativa para o ensino de ciências nos anos iniciais. Revista Contexto \& Educação, 33(104), 365-394.

Freitas, M. D. C. S. D., Fontes, G. A. V., \& Oliveria, N. D. (2008). Escritas e narrativas sobre alimentação e cultura. EDUFBA.

Ferreira, I. S. C. (2020). Avaliação dos hábitos alimentares de uma amostra da população portuguesa durante o período de contenção social no combate à pandemia Covid-19. 
Research, Society and Development, v. 10, n. 15, e129101522475, 2021

(CC BY 4.0) | ISSN 2525-3409 | DOI: http://dx.doi.org/10.33448/rsd-v10i15.22475

Firmo, M. C., Cunha, G. R. (2020). A influência da tecnologia de aplicativos de delivery no comportamento do consumidor. Os direitos humanos na era da tecnologia, (I), 73-80.

Junior, E. R., Junior, P. V. C., Flavio, L. F., \& David, M. F. (2019). A gourmetização do hambúrguer. Revista Valore, 4(1), 923-935.

Junior, L. C. L. (2020). Alimentação saudável e exercícios físicos em meio à pandemia da Covid-19. Boletim de Conjuntura (BOCA), 3(9), 33-41.

JÚNIOR, L. A. T. L. (2019). Hamburguer: Um bled de carnes e redução de tangerina. Revista de Gastrronomia., 1(1).

Moratoya, E. E., Carvalhaes, G. C., Wander, A. E., \& Almeida, L. M. D. M. C. (2013). Mudanças no padrão de consumo alimentar no Brasil e no mundo. Revista de Política agrícola, 22(1), 72-84.

Marques, D. P. (2019). Qualidade percebida e continuidade de utilização de apps de food delivery (Doctoral dissertation, Instituto Superior de Economia e Gestão).

Melo, E. S., Macedo, D. L., \& Calazans, S. (2018). Gestão de custos em serviços de alimentação coletiva: uma revisão sistemática. REVISTA UNI$R N, 16(1 / 2), 110$

Moraes, J. M. M. (2017). Porque as pessoas comem o que comem? Comparação das motivações para comer entre dois contextos socioeconômicos díspares no Brasil (Doctoral dissertation, Universidade de São Paulo).

Morais, C. (2005). Escalas de medida, estatística descritiva e inferência estatística.

Panizzon, M., De Toni, D., Larentis, F., \& Milan, G. S. (2013). A percepção da imagem de preço em serviços: um estudo de abordagem quantitativa sobre os cursos de lato sensu de uma Universidade. REAd. Revista Eletrônica de Administração (Porto Alegre), 19, 646-674.

Perry, K. S. (2004). Queijos: aspectos químicos, bioquímicos e microbiológicos. Química nova, 27, 293-300.

Piana, M. C. (2009). A construção do perfil do assistente social no cenário educacional.

Ponte Neto, E. D. S. (2016). I Food: um estudo sobre o comportamento de compra do consumidor.

Reis Verticchio, D. F., \& de Melo Verticchio, N. (2020). Os impactos do isolamento social sobre as mudanças no comportamento alimentar e ganho de peso durante a pandemia do Covid-19 em Belo Horizonte e região metropolitana, Estado de Minas Gerais, Brasil. Research, Society and Development, 9(9), e460997206-e460997206.

Ribeiro, Í. A. P., da Rocha, M. O., Cunha, D. C. L., da Silva Araújo, A., Amaral, I. N., Marques, L. L., \& Cunha, M. B. (2020). Isolamento social em tempos de pandemia por Covid-19: impactos na saúde mental da população. Revista Enfermagem Atual In Derme, 92(30).

Romans, J. R., Costello, W. J., Jones, K. W., Carlson, C. W., \& Ziegler, P. T. (1985). The meat we eat. The Interstate Printers and Publishers, Inc.

Santos, C. R. A. (2006). O império mcdonald e a mcdonalização da sociedade: alimentação, cultura e poder.

Silva, M. A. D. (2018). Fermentação natural-conhecendo o levain e sua aplicação comercial no mercado de Fortaleza.

Souza, A. D. M., Pereira, R. A., Yokoo, E. M., Levy, R. B., \& Sichieri, R. (2013). Alimentos mais consumidos no Brasil: Inquérito nacional de alimentação 2008-2009. Revista de Saúde Pública, 47, 190s-199s.

Stecca, R. D. S. (2020). Gestão da informação e inteligência competitiva aplicadas ao serviço delivery online do setor de alimentação.

Veiga Neto, A. R., \& Melo, L. G. N. S. D. (2013). Fatores de influência no comportamento de compra de alimentos por crianças. Saúde e Sociedade, 22(2), 441455 .

Walther, B., Schmid, A., Sieber, R., \& Wehrmüller, K. (2008). Cheese in nutrition and health. Dairy Science and Technology, 88(4-5), 389-405.

Zielke, S. (2010). How price image dimensions influence shopping intentions for different store formats. European Journal of Marketing. 